\title{
Situating organizational action: the relational sociology of organizations
}

\author{
Alistair Mutch, Rick Delbridge and Marc Ventresca
}

\section{Introduction}

This special issue seeks to address what we feel is a growing concern with the nature of theorizing about organizations and, in particular, how we understand and situate organizational action. Our concerns are threefold. First is the concern that many of the accounts which centre on process and practice tend to downplay the importance of the broader settings in which such action takes place. Second is the worry that approaches such as new institutionalism tend to downplay the role of agency. More broadly, we have an increasing unease with the easy eclecticism - the mixing and muddling - of distinct and complex ideas and concepts that has become increasingly commonplace in organization studies. These are issues that lie at the heart of sociological theorizing more generally and the position we outline below draws on the work of a number of social theorists. The arguments we advance resonate in important ways with the points of view developed by Emirbayer (1997) in his 'manifesto for a relational sociology'. Specifically, we share his focus on ontology, his emphasis on the primacy of contextuality and process in sociological analysis, an attention to causal explanation that seeks to avoid both pure voluntarism and structural determinism, a requirement for theoretical consistency across levels of analysis and an advocacy of evaluation and internal debate around the thematization of issues and problems in order to facilitate theory building within and across traditions. 
In this paper we advocate a relational form of analysis and tease out what such an approach might mean for organization studies. This draws our attention to a number of connections that we outline in this introduction. In particular, we wish to emphasize some promising approaches that are echoed in the papers that follow, all of which share a commitment to a relational form of analysis. Some of this work, notably that of Bourdieu, is receiving more attention in mainstream debates (Ozbilgin and Tatli, 2005). However, this is often in a way, we would suggest, that tends not to deal with the detail of the approach but with some headline features. One argument that we present in this introduction, therefore, is the need to engage in a serious fashion with the full extent of the bodies of work that we highlight here rather than a partial and selective re-presentation of parts of a wider whole. To do so will help researchers to recognize the nuances, variations and possibilities that lie within these.

The connections we outline run in a number of directions. At one level there are the connections between organizations and their wider world. The concern to draw such connections, to recognize that organizations do not somehow float in a neutral 'environment' but that their actions produce and reproduce the world that they inhabit, has been behind the use of terms such as 'organizational field' by some within new institutionalist approaches. Some would argue that the full import of such usages is not explored and that their proper investigation would lead to a useful debate with those heading, as it were, in the opposite direction, that is, from the outside of organizations inwards (Lounsbury and Ventresca, 2003). In the comparative business systems perspective, for example, there are suggestions that firmer connections are needed between broad institutional patterns discernible at national or regional level and the actions that occur within and between organizations. Whitley (2003), for 
example, has recently suggested that the work of critical realists like Margaret Archer which we discuss below may be of value here.

This latter suggestion points to the existence of another set of connections that we have anticipated above - those between organization theory and wider sociological and social theorizing. That the bulk of theorizing organization now takes place in business schools may well have contributed to the tendencies toward both a fragmentation of the discipline and a narrowing of focus in the resulting attenuation of the links between organization theory and broader forms of theorizing. We focus in the remainder of this introduction on three sets of approaches, all of which hail from outside the business school but all, we think, of considerable potential in revitalizing organization theory and rebuilding connections. We also have the task of showing that there are some interesting points of connection between these three approaches. For example, and importantly, they each share a depth as well as breadth to their conceptualization of relations (i.e. they share a stratified ontology). To show points of connection, however, does not mean to erase or elide differences. We present these three approaches not as some sort of unified 'answer' to the problems of situating organizational action, but rather as promising frameworks which need further debate and discussion (in the spirit of Delbridge and Ezzamel, 2005).

The three approaches we wish to explore in a little more detail share a location in the domain of sociology and social theory. The first, relational pragmatics, is the most clearly sociological in location and is derived from American pragmatism and its engagement with a number of European bodies of work. One of these constitutes the second approach that we wish to consider, that inspired by the work of the French 
social theorist Pierre Bourdieu. Bourdieu was concerned with a wide range of social phenomena but not, until the latter stages of his work, with organizations directly. However, some of his terms, such as 'field', have been picked up and deployed within approaches such as new institutionalism. The concern of some is that in the process they have been divorced from their wider context in Bourdieu’s schema and so shorn of explanatory power. This is a question which we return to later. The third set of approaches are those based on critical realism, notably the morphogenetic approach of Margaret Archer $\stackrel{i}{*}$

It is important here for us to distinguish between critical realism as a philosophical approach and the substantive theories that make use of it. Reed (2005a: 1632) notes that critical realism redefines the 'explanatory task' in organizational analysis and impacts on at least three key issues or dilemmas in social theory: the structure/agency problem, the status of, and relationship between, historical, structural and discursive analysis in social science, and in explaining social change. In some ways, therefore, critical realism forms an underlying thread that can tie together our discussion of substantive theories that otherwise have significant differences in approach. In our discussion of these differences we will pay rather more attention to critical realism as a way of sorting out underlying issues of ontology that might help in furthering the connections that we identify. We consider a number of areas in greater depth.

The first is the commitment of all three approaches to a realist ontology and it is here that we present a little more material on critical realism, material which also relates to current debates over, and interest in, the development of 'scientific realism' (a further connection that we could make). Building on this, we explore the commitment that all 
three approaches share to a relational perspective on social phenomena. This leads us into a closer discussion of the nature of the relationship between agency and structure that the three approaches suggest. This area is by no means agreed between the three, but there are points of contact that suggest useful ways forward.

The relational approach that is supported emphasizes the value of time in constructing analyses of concrete organizational developments, which leads us into some consideration of methodological issues. The emphasis here on the need to relate methods to the nature of the question being asked and to revise them in the course of investigations is clear. We consider each of these areas in a little more detail before suggesting some areas which still need further debate and clarification. We do not attempt a formal reconciliation between these three approaches, for that would be impossible in the space available (and probably unproductive), but we hope that by drawing these connections we can encourage others to pursue their many ramifications in what we feel would be a productive endeavour for those concerned with the relationship of organizational actions to the wider world.

\section{Ontological foundations}

We start, then, with a brief review of the ontological commitments of our three approaches. For those explicitly based on critical realism, this is straightforward, but the approaches of both Bourdieu and the relational pragmatists also seem compatible with a broadly realist ontology. Whilst this discussion might not be present in the work of Bourdieu himself, commentators have suggested that his ontological presuppositions are best characterized as realist (Fowler, 1997: 6). As there is considerable interest with some parts of organization theory in notions of scientific 
realism (McKelvey, 2003) it is worth at this point considering the nature of critical realism and its relation to substantive theorizing in a little more detail.

It is important at the outset to be clear about the claims being made by critical realism about the 'truth', particularly because some seem to align it with a search for an 'absolute truth’ (Inns, 2002). Such claims are often made in passing with little sign of engagement with the literature, but they continue to pollute the terms of the debate. Referring to the work of Pinder and Bourgeois (**Alistair ref?), for example, Inns writes that they 'represent the most extreme position of the critical realist strand, ignoring the arguments from, for example, Lakoff and Johnson (1980) that it is impossible to distinguish between literal and figurative language as ostensibly literal language turns out on closer inspection to be figurative' (** Alistair - page number? )

What the position adopted by Pinder and Bourgeois actually represents is one much closer to the 'scientific realism' that we discuss further below (and one which ignores the realist position that Lakoff and Johnson espouse, in which metaphor is related to our embodied engagement with the world). For what one notes in the critical realist position is a much more tentative approach to issues of truth and reality than suggested by some critics. The focus is on ways of understanding and explaining the world whose results are always avowedly provisional and corrigible, but which are presented as outcomes subject to further adjudication and debate. This is not the world of grand narratives, but it is one in which positions are taken seriously. The focus is on the need for clarity of exposition and on the sincere and committed engagement with the ideas of others. The question of truth is perhaps nicely summed up by Hilary Rose when she observes that '[p]erhaps truth in the strong sense used by Rorty et al. 
never exists outside the certainty of 'true forme', which I get when I read a poem or a novel' (Rose, 1994: 25). What is central to critical realism, then, is not a claim to a privileged access to the truth, but a genuine attempt to formulate better means of understanding.

In such means of understanding there is, of course, the fundamental metaphysical assumption that there is a world independent of our imagining of it. We cannot have any direct and unmediated access to this world, but we have not to tangle up our conceptions of the world with the existence of the world. Working from this initial premise, Bhaskar (1979) was concerned to explore what properties the world must possess for science to be possible. On this premise, he distinguished several levels of reality.

On the surface are empirical events, those which are accessible as sense data and which form the basis of 'commonsense' or 'naïve' realism. Below these events is the actual, often accessible only through indirect means, such as the scientist's electron microscope. However, this 'scientific realism' is not the full extent of the real, nor does it explain what scientists do, a position which might be contrasted to that which seems to be adopted in the 'mainstream' of organizational analysis (Boal, Hunt \& Jaros, 2003; McKelvey, 2003). Rather, argues Bhaskar (1979), the real is to be found in the causal mechanisms that produce the event states that we record as the 'actual' and it is these causal mechanisms that scientists are concerned to identify and explore. These mechanisms might only operate within a particular range of constraints, and there may be other mechanisms that work to confound their operation. That is, 
mechanisms may exist but not be activated, or their effects might be hidden by countervailing tendencies.

Reality is also stratified and emergent. These twin concepts are nicely illustrated in the work of the biologist Stephen Rose (1993), whose explorations of the making of memory are located in a framework informed by Bhaskar's work. The mind emerges from certain physical and biological properties, but it cannot be reduced to them. That is, a particular combination of physical and chemical properties gives rise to the biological apparatus of the brain, but this working cannot be reduced to a simple collection of individual parts. In turn, Rose's experiments indicate that memory organizes as a self organizing system that depends on biological substances to operate, but cannot be reduced to any one part of this system. This allows Rose to resist the claims of genetic determinism and further allows us to posit the notion of the social as a distinct layer, dependent on the actions of people but not reducible to individuals. A key concept here is the notion of 'emergent properties', the idea that levels possess properties that are sui generis. Finally, the difference in levels also corresponds to an increasing openness and complexity. At some levels we may be able to attain closure, or it may be feasible for scientists to attain such closure through controlled experimentation. This allows for prediction, but in open systems there is increasing contingency which makes prediction problematic; laws give way to tendencies. At the level of human systems, all systems are open and further complexities need to be added. We have the operation of the 'double hermeneutic', that is, not only do we have to interpret the results of our observations, but our research subjects produce their own readings of the world (Outhwaite, 1987). Sometimes these readings incorporate our own and this makes the 'scientific' 
isolation of variables a perilous business. This raises the central issues of contingency and free will. These make prediction fraught with difficulty and suggest that a more modest goal of explanation is appropriate for those exploring human activity.

It is important that critical realism has not sought to separate off the works of natural and social science. There are the twin dangers, especially in more interpretivist traditions, of either ignoring the natural sciences and hence ceding a large part of the argument, or seeking to deny the methods and achievements of the natural sciences. Indeed, Bhaskar himself recognized the applicability of some of these ideas emerging from the philosophy of science in formulating his Transformational Model of Social Action. However, those ideas have in turn been critiqued by those from within the critical realist tradition, such as Archer, who are more familiar with broader social theorizing. The important point here is that critical realism is compatible with a wide range of substantive theories (Collier, 1994). There are no 'master keys' in critical realism but rather a commitment to act as a philosophical 'under-labourer' for the social sciences. The notions of the search for causal mechanisms and the emergence of phenomena over time are both important in the discussions that follow. The importance of time will be considered in more detail later, but it does form an important part of a commitment to relational forms of analysis.

\section{Relational analysis}

This commitment to a relational form of analysis is foregrounded in the work of Mustafa Emirbayer. Building on the work of Dewey in particular, but referencing a wide range of other authors, he suggests that we need to see 'relations between terms or units as pre-eminently dynamic in nature, as unfolding, ongoing processes rather 
than as static ties among inert substances'- (1997: 289). This is elaborated in his discussion of causality which recognizes that social actors are embedded in space and time and respond to specific situations in ways that are not captured in accounts which reify structures as causal factors. In taking an action orientation, however, Emirbayer (1997: 308) argues that a tendency to pure voluntarism must be avoided 'by insisting upon a search for robust explanatory processes that operate across a multiplicity of social situations'. This position clearly shares a great deal in common with that taken by critical realists such as Archer to which we return shortly.

In the course of developing his argument for a relational sociology one of the authors Emirbayer draws on is Bourdieu, who has also championed a processual, relational view of the sociological enterprise. His focus has been on the emergence of order from practice, not through commitment to pre-established goals but emergent from the flow and flux of practice. There is, he argues 'an economy of practices, a reason immanent in practices, whose 'origin' lies neither in the 'decisions' of reason understood as rational calculation nor in the determinations of mechanisms external to and superior to the agents' (Bourdieu, 1990: 50). However, this commitment to processual analysis is dependent in turn on the centrality of the notion of habitus in imparting regularity to the outcomes of practice.

For Bourdieu, habitus is the crucial link between structure and agency, but it is one that DiMaggio (1997) suggests may place too great an emphasis on experiential learning and so underplay the extent of other factors in shaping culture. In particular, he points to the promise of the notion of institutional logics as outlined by Friedland and Alford (1991). There are interesting links here to the conceptualization of agency 
that Archer (2003) presents. These links work in two ways. One is the stress that Archer (1996) places on the relations between systems of ideas and actions that open up spaces for agential intervention. The second is the insistence on the emergence of agency from, but not its reduction to, embodied personhood (Archer, 2003). This allows for the integration of the insights from studies of cognition that DiMaggio (1997) draws attention to. However, the mobilization of such insights rests in part on a clear engagement with the central question of the nature of the relationship between agency and structure.

This question lies at the heart of debates over the lack of attention to agency and interest with new institutionalism, as recognized by, for example, DiMaggio (1988). The theorists called in most frequently to repair the damage are Bourdieu and Giddens. Part of our problem, however, is the manner in which ideas are drawn from such thinkers in a way that both abstracts them from their broader context and misrecognizes key aspects of their use. The entry of Giddens via DiMaggio’s reading of Bourdieu, for example, perhaps explains the persistent use of 'structuration' to refer to the structuring of a field that does not seem to derive from Giddens' use of the term (Scott, 2001). In turn the notion of the 'field' seems to be taken from Bourdieu, but in a fashion that abstracts it from its relationship to other core concepts like capital and habitus. These concepts need to be seen as part of a broader endeavour. That endeavour is to overcome what is seen to be the false dualism between agency and structure; in the words of Wacquant 'not simply to combine, articulate or join agency and structure but, more fundamentally, to dissolve the very distinction between these two seemingly antinomic viewpoints of social analysis’ (Waquant 1993:3). 
This characterization of Bourdieu places him close to the 'mutual constitution' approach essayed by Giddens and, as such, in considerable opposition to the ideas of Archer that we examine further below. It is, therefore, worth explaining why we explore these ideas in more detail. Part of the reason is that it was Giddens on whom Bhaskar drew to develop his model of social action. But a more important reason is that it is Giddens who is most frequently drawn upon in a wide range of organization theory whenever it is that the wider context needs to be drawn in. However, the manner in which this is done often uses the ideas more as a rhetorical device in pointing to a recognition of a relationship rather than as an analytical device. This is sometimes done in a way that recognizes some of the problems that we discuss below but chooses to sidestep them. Here is what Barley and Tolbert (1997: 99) have to say:

Although the critics of structuration theory have aimed their critique at problems they believe to be inherent to the theory's logic and, for this reason, have sometimes argued for re-establishing the separation between structure and action that Giddens sought to transcend [citing Archer], we submit that the worth of the critique actually lies in the epistemological rather than the ontological issues that it raises.

Actually, when one reads the account that Barley and Tolbert present it is possible both to argue that their version of Giddens' structuration theory seems divorced from the original and that, in practice, their account would be more congruent with Archer's (1995) ideas. We can note similar fudging of these key questions elsewhere (Hendry, 2000; Heracleous and Hendry, 2000). The problem is that we need to be clear about the differences, as they lead to different logical entailments. Once again, 
we return to our point earlier about the need for clarity and consistency in the use of concepts. We can see some of the problems involved through a closer examination of Archer's critique of Giddens' approach, a critique that is shared and drawn upon by Emirbayer and Mische (1998) in their development of notions of agency.

\section{Conceptualizing action}

For Archer (1995), those analysts who privilege the impact of structures on agents are 'downward conflationists', in which actions are simply an epiphenomenon of deep structures. On this reading, once we have acquired the key to the structures, we can simply 'read off' the correspondent action. By contrast, those who focus on human interaction, such as the Symbolic Interactionists, are ‘upward conflationists' for whom society is simply an aggregation of a myriad of individual interactions, with no discernible shape or pattern beyond these. Archer rejects both (as does Giddens) but finds that Giddens' structuration resolves the tension by dissolving it altogether. Her solution, by contrast, is to insist on the importance of both agency and structure and to suggest that what is important is to explore the relationship between the two. This is the core of what she terms her 'morphogenetic' approach ('The 'morpho' element is an acknowledgment that society has no pre-set form or preferred state: the 'genetic' part is a recognition that it takes its shape from, and is formed by, agents, originating from the intended and unintended consequences of their activities’ (Archer, 1995: 5)), which she formulates as follows:

every morphogenetic cycle distinguishes three broad analytical phases consisting of (a) a given structure (a complex set of relations between parts), which conditions but does not determine (b), social interaction. Here, (b) also 
arises in part from action orientations unconditioned by social organization but emanating from current agents, and in turn leads to (c), structural elaboration or modification - that is, to a change in the relations between parts where morphogenesis rather than morphostasis ensued. (Archer, 1995: 91)

Such an approach draws on the notion of emergent properties, derived from Bhaskar's work explored above, to argue that whilst society is quite clearly the product of human activity, it is not necessarily the product of those humans 'here present'. That is, the previous activities of human actors create structures (institutions, roles, routines) that then both constrain and enable actors in the next round of activity. In each cycle, actors begin their interactions in a context which they did not create but which enables certain outcomes and makes others unlikely or difficult.

To label them as unlikely or difficult does not, however, make them impossible. Agency is not determined by context, but has to take into account opportunity cost in exercising choice. Such choice, of course, may well not appear as such, with certain courses of action being practically unavailable in many contexts. However, the result of social interaction is structural elaboration, either change or stasis. It is down to empirical analysis to determine the content of each cycle, but a key part of such analysis will be an adequate exploration of the formative context. Time is therefore a critical dimension of this approach, as we will discuss shortly.

Archer's development of critical realism, therefore, preserves a strong commitment to a relational form of analysis in which agency and structure have to be held apart to explore the nature of both, and the relationship between their infolding development 
over time. This commitment is shared by Emirbayer and Mische (1998) in their discussion of the nature of agency. Whilst their focus is on agency, this is quite clearly developed in relation to structures; for them it is:

the temporally constructed engagement by actors of different structural environments - the temporal-relational contexts of action - which, through the interplay of habit, imagination, and judgement, both reproduces and transforms those structures in interactive response to the problems posed by changing historical situations. (Emirbayer and Mische, 1998: 970)

It is worth discussing the characterization of agency that both they and Archer derive in order to contrast it with that presented by Bourdieu, for some interesting connections emerge. Emirbayer and Mische suggest three dimensions to agency interational, through an orientation to the past, practical evaluative, through an orientation to the present, and projective, through an orientation to the future - that are derived from their engagement with, in particular, the work of Mead. They suggest that much of the work in sociology has been concerned with the role of habit in conditioning agency, and they note both Bourdieu and Giddens in this regard as stressing this component. In their desire to suggest that present and future orientations also need consideration, they draw on Mead's conception of the 'internal conversation'. This is where an actor reflects upon their engagement with existing structures and can be the source of change.

Interestingly, Archer's (2003) most recent work also draws on this idea and develops the notion of the internal conversation as the key mechanism that bring actors into 
collision with structures. She suggests is that it is not good enough to simply outline a relationship between agency and structure; what we also need is an account of change. This, she argues, leads us to question why it is that some seek change, when others, placed in the same contexts, are content to accept existing arrangements. In this endeavour she places considerable emphasis on the delineations of forms of reflexivity. In contrast to, for example Giddens (1990), whose account of reflexivity stresses the knowledgeability of all actors in modernity, Archer suggests that there are different forms of reflexivity, based on the form of the internal conversation that is adopted. We all, she argues, conduct such conversations and use them to monitor and evaluate our fundamental moral projects, but we do this in different ways shaped by our involuntary positioning in social contexts.

For some (the majority in her exploratory study), the internal conversation needs to be completed in the context of others. Concerns, that is, have to be verbalized and shared with others in order for resolution to be obtained. This group are the 'conversational reflexives' and their engagement with the world is characterized by measures to maintain continuity of context. In this they will tend to avoid contact with structures or work 'with the grain', in sharp contrast to the 'autonomous reflexives'. The autonomous reflexive completes their own internal conversation in relative (and these terms are all relative) isolation from the concerns of others. This has the potential to bring them into conflict with and seek to change the structures which surround them. This feature is shared to some extent by the third category, that of the 'meta reflexive'. The meta reflexive uses the internal conversation not only to monitor personal projects but also to reflect upon the process of reflection itself (thus more closely approaching Giddens' conceptualization of reflexivity). This does not 
necessarily lead to broader change, however, so much as to the dissatisfaction of the person with the nature of the world and their efforts in it. The final category is that of the 'fractured reflexive', the person who, for some reason, never acquires the ability to conduct a satisfactory internal conversation. These are society's victims, never able to achieve their personal projects and remaining in the position of what Archer would term 'primary agents', that is, with their life chances determined to a significant degree by their involuntary positioning.

The approaches of both Archer and Emirbayer and Mische to agency stand in considerable contrast to that essayed by Bourdieu. As we have seen, his focus on the importance of habitus, a set of durable dispositions to act that are transposable across contexts, tends for some critics to be stronger at explaining continuity rather than change (DiMaggio, 1997). By contrast, the approaches of both Archer and Emirbayer and Mische suggest orientations to both stasis and change. There is not the space here to attempt to map the two sets of ideas to each other, but this might be a fruitful endeavour. Archer's categories, for example, might suggest why some adopt more projective forms of agency and hence, the roots of broader change. Both suggest the importance of time in organizational analysis, something shared by Bourdieu. These commitments have both ontological and methodological significance. The focus on emergence over time in Archer is central to the possibility of relational analysis, in shaping a world in which the contexts of action are given to those who have to take action within their constraints.

Whilst we have pointed to some differences between the three approaches, a common link is the injunction that we have to pay due regard to the importance of time in our 
analyses (Reed, 2005a, b). For Archer and others basing their ideas on critical realism, the key task is the construction of analytical narratives that explore situations over time with a view to uncovering causal mechanisms. Her earlier work on educational systems, for example, draws on a period of several hundred years to outline contrasts between different configurations, configurations which then shape distinctive patterns of action (Archer, 1979). For Emirbayer and Mische too time is central, given the definition of structures as 'temporal-relational contexts of action'. And the historical development of fields is absolutely key to Bourdieu's enterprise. His work on the importance of mental habits, particularly in fields of artistic endeavour, draws on sources such as Panofsky's (1957) analysis of the links between medieval scholasticism and architecture.

\section{Methodological concerns}

The depth of historical understanding contained in the work of Archer and others, of a sense of context which is not something shaped in the past decade but rather over centuries, has been key to the development of a relational approach. This poses a problem for organizational scholars, oriented as they often are, partly through institutional pressures, to cross-sectional snapshots of time. This is not just a question of orientation or skills, but is often one of time and the availability of materials. It suggests that inter-disciplinary efforts are badly needed and that attempts to stimulate historical enquiry as a central part of organizational analysis (such as the recent journal Management and Organization History) are to be encouraged.

Another methodological injunction can be derived from Bourdieu, that of the need to revise approaches as research projects develop. That is, the importance needs to be 
placed on the questions to be addressed, not the methods employed. In this, we can get some support from critical realism. Drawing on the work of Andrew Sayer, we can contrast the 'ontological boldness' of critical realism with its more relaxed approach to issues of epistemology. That is, while critical realists make bold claims about the nature of reality they are altogether more cautious in their epistemological claims. As Sayer argues, critical realism

accepts 'epistemic relativism', that is the view that the world can only be known in terms of available descriptions or discourses, but it rejects 'judgemental relativism' - the view that one cannot judge between different discourses and decide that some accounts are better than others. (2000: 47)

This means that critical realism is compatible with a wide range of methods, the crucial issue being the nature of the question to be addressed. That is, critical realists start by clarifying their ontological assumptions about the world and then proceed to the selection of methods. This means, for example, that there can be a place for statistical approaches, although, as Sayer cautions, 'they are primitive tools as far as explanation is concerned' (1992: 198). The key problem, however, for Sayer is the way in which a focus on technique hinders an exploration of underlying assumptions. Once again, we return to the centrality of ontological questions.

However, this focus on ontology and a concomitant requirement for the systematic articulation of philosophical commitments and explanatory logics is something which is disquieting to many of those who might otherwise find several of the ideas espoused here of value. This seems to lie behind claims that critical realism, for 
example, is an 'imperialist' enterprise which suggests that it has the master key to unlocking the mysteries of organizational life (see the debate between Reed, 2005a, b and Contu and Willmott, 2005). Quite clearly here, we have to strike a balance between the advocacy of a perspective because it seems to us to contain much of value and a measured approach to the gains to be made.

Part of such an approach rests in recognizing the limitations that we outline above about the relationship between these forms of enquiry and the 'truth'. The relationship is one of striving towards better forms of understanding whilst always recognizing that such attempts are provisional and subject to revision. An alternative concern is, by contrast, the notion that the ideas we are outlining are nothing new; that, in the words of Howard Aldrich (1992: 22) on new institutionalism, they are 'just plain sociology'. Not only is there very little that is new, but a cause for concern is the way that previous analyses are retro-fitted into a particular category (Watson, 2006). In many senses this criticism is true, but it perhaps misses the mark. The concern is often that, whilst the ideas may seem nothing new, they are regularly ignored in contemporary forms of analysis, which seem to be fated to make the same mistakes because they ignore the rich legacy of 'plain sociology'. One would want to draw a distinction here, difficult though it is, between the uses made of a body of ideas and the logical entailments contained in that body of ideas. The problem with many of the critiques of, say, critical realism is that they pick on one or two particular instantiations of the ideas and base their criticism on these, failing to recognize that such bodies of ideas are dynamic (Mutch, 2005) and contain substantial differences within them (Reed, 2005b). It is often the case that the criticisms are also ones which are the subject of debate within the body of ideas. Again to echo Emirbayer (1997), 
these are not bodies of dogma to be followed slavishly, but rather living sets of ideas to be debated, refined and evaluated and whose value lies in the purchase that they give us on understanding the world. That they can be applied in different ways can be seen in the collection of papers that follows.

\section{The papers}

We start with two papers both seeking to develop aspects of the new institutionalist project by drawing on new sets of theoretical resources. For both the focus is the debate around the notion of the 'institutional entrepreneur', which has been seen as a key means of addressing DiMaggio's (1988) critique of the lack of attention to questions of power, interest and intentionality. Bernard Leca and Philippe Naccache draw on critical realism to build a 'non-conflating model of institutional entrepreneurship'. They argue that critical realism allows them 'to move beyond actors' discourses, decipher between context and structural mechanisms and highlight hidden power mechanisms.' In turn they suggest that 'recent developments in institutional analysis allow us to flesh out abstract notions of critical realism, such as 'structures", in this fashion demonstrating the value of mutual engagement between these two traditions. Interestingly, their conclusions also suggest that there may be merit in further drawing on Bourdieu, a theorist who supplies the key resources drawn upon in Julie Battilana's paper. She is also concerned with the paradox of 'embedded agency' present in the institutional entrepreneurship debate and seeks to build an account that more rigorously delineates the spaces for action generated by social positions. In drawing on Bourdieu she eschews the frequent focus on habitus in favour of his notion of the field. She suggests that the merit of such an approach is that it sets up the 'micro foundations for the development of a theory of institutional 
entrepreneurship'. It also, she suggests, opens up some promising lines of inquiry that we could relate to the notions of agential orientation that we alluded to above in drawing on the work of both Archer and Emirbayer and Mische, and which also come out in one of our other papers.

This next pair of papers illustrate two contrasting approaches to the application of broader institutional perspectives to particular domains. They both feature a detailed focus on particular subject domains but the direction of the approach is different in each. For Steve Fleetwood and Anthony Hesketh the move is in from a detailed specification of a critical realist meta-theory to assist in the development of domain specific concepts in understanding the debate over the putative link between human resource management and organizational performance. They use the resources of critical realism to engage in a detailed critique of existing positions, showing how such positions confuse empirical correlations (or lack of them) with a properly causal account. They suggest that critical realism, by supplying them with ontological clarity, is a more fruitful approach. Interestingly, they suggest that attention needs to be paid to what they term 'reflexive performance', something which they illustrate through extensive quotes from practitioners. By contrast, Paul Edwards, Monder Ram, Sukanya Sen Gupta and Chin-ju Tsai seek to build from within extensive work on small firms, and in particular employment relations within such firms, to develop a framework from analysis drawing upon the existing strengths of an institutionalist approach. They argue that whilst there has been much fruitful empirical work a more structural framework is needed to build explanatory accounts. They develop such a framework drawing upon extensive work in 'low value added' firms, a category which they argue 'provide a useful context in which to examine the interplay of 
different domains.' The importance of their framework, they suggest, 'is its underlying logic of giving shape to the many powerful insights of institutional theory'.

Renate Meyer helps to remind us of some of those insights in her valuable review essay, which also has value in opening up areas of literature and research likely to be unfamiliar to many with access only to English language material. For example, she notes that in the collected essays of Luckmann that she reviews there is a clear focus on power and suggests that '[i]f we do not pay enough attention, it is our research foci that distract us and not the framework'. This is a powerful reminder that all too often our views of a particular approach are taken through a chain of interpretation that frequently results in the misattribution of positions. Meyer's engagement with this literature also points to some interesting observations about the nature of the institutional entrepreneur, something which is an important sub-theme in our papers. She warns us in particular about the dangers of meaning attribution based on retrospective reflection: 'we need', she suggests, 'to differentiate carefully when assuming that these actors strategically pursue their interests'. This is a welcome reminder, as are some intriguing notes on methodology. In particular, one notes with interest the recommendation for 'artificial stupidity and slowness' in hermeneutic approaches; this move accords with the need for the reflexive monitoring and changing of research approaches that we find in Bourdieu but conflicts with the demands to publish speedily that are institutionalized in many contemporary research settings. 
These papers, then, suggest a number of different approaches to the institutional shaping of organizational action. They all suggest valuable ways of reshaping contemporary debates in a number of domains. One challenge for our authors has been to combine theoretical rigour with empirical illustration, something which is extremely challenging in the context of the space available in journals. One critique of approaches such as critical realism is that they are long on theoretical exegesis, short on empirical application. Whilst fair in a number of cases, such a criticism in its turn fails to pay due attention to the material conditions of publication. Several of our papers point to supporting work published elsewhere and it is important that we judge work across the full range. However, such a strategy also points to the argument that it is perhaps better and more valuable to develop arguments with a combination of theoretical and empirical work at book length. A powerful recent example is Tony Elger and Chris Smith's work on Japanese firms and their 'transplantation' into the United Kingdom. The fruits of a decade of detailed work employing a range of methods - comparative case study analysis, observations, in-depth interviews - this deploys a specifically critical realist framework which builds on work in a number of traditions to 'identify the fundamental social relations and processes that underpin and condition the specific institutional patterns and organizational practices that characterize the evolution of competing capitalisms and competing firms' (Elger and Smith, 2005: 68). At the same time they stress both collective and individual agency in a way which can best be displayed when there is sufficient space to elaborate on the details of contest, negotiation and accommodation. Our authors have not had such a degree of space but we thank them for their collective efforts in demonstrating the continuing value of the search for more helpful ways of situating organizational action. 


\section{Concluding remarks}

In this introduction we have advanced a relational sociology of organizations that seeks to address a number of key concerns with the way in which organization studies has been developing. We have advocated an historically and institutionally grounded approach which both recognizes and explores the constraining and enabling character of social structures and the prospects of agency on the part of social actors. We acknowledge that this is far from novel but it does require us to make explicit our ontological assumptions and to engage with various methodological concerns.

Our more specific purpose has been to identify, describe and begin to build connections between related but largely independent bodies of work that provide potential insights for our understanding of social phenomena. In this we have been very keen to avoid, and to argue explicitly against, the easy eclecticism or ad hoc reasoning that too often characterizes the selective (mis)representation of various social theories in their application in organization studies. The development of the field of organization theory will benefit from the self conscious and reflexive engagement and debate both within and across our various research positions and traditions only if such debates are conducted on the basis of holistic evaluations and interpretations that recognize (and value) difference.

\section{References}


Aldrich, H. (1992) 'Incommensuable paradigms? Vital signs from three perspectives', in M. Reed and M. Hughes (eds.), Rethinking Organization, London: Sage, $17-45$.

Archer, M. (1979) Social Origins of Educational Systems, London: Sage.

Archer, M. (1995) Realist Social Theory: the morphogenetic approach, Cambridge: Cambridge University Press.

Archer, M. (1996) Culture and Agency: the place of culture in social theory, Cambridge: Cambridge University Press.

Archer, M. (2003) Structure, Agency and the Internal Conversation, Cambridge: Cambridge University Press.

Barley, S. and Tolbert, P. (1997) 'Institutionalization and structuration: studying the links between action and institution', Organization Studies, 18(1) 93-117.

Bhaskar, R. (1979) The Possibility of Naturalism, Hemel Hempstead: Harvester.

Boal, K. B., Hunt, J. G. and Jaros, S. J. (2003) 'Order is free: on the ontological status of organizations', in R. Westwood and S. Clegg (Eds.) Debating Organization, Oxford: Blackwell, 84-97.

Bourdieu, P. (1990) The Logic of Practice, Cambridge: Polity.

Collier, A. (1994) Critical Realism: an introduction to the philosophy of Roy Bhaskar, London: Verso.

Contu, A. and Willmott, H. (2005) 'You spin me round: The realist turn in organization and management studies', Journal of Management Studies, 42(8), 1645-1662.

Delbridge, R., and Ezzamel, M. (2005) 'The strength of difference: Contemporary conceptions of control', Organization, 12(5), 603-618. 
DiMaggio, P. (1988) 'Interest and agency in institutional theory', in L. Zucker (ed) Institutional Patterns and Organizations: Culture and Environment, Cambridge: MA: Ballinger 3-19.

DiMaggio, P. (1997) 'Culture and cognition', Annual Review of Sociology, 23 263287.

Elger, T. and Smith, C. (2005) Assembling Work: Remaking Factory Regimes in Japanese Multinationals in Britain, Oxford: Oxford University Press.

Emirbayer, M. (1997) 'Manifesto for a relational sociology', American Journal of Sociology, 103(2), 281-317.

Emirbayer, M. and Mische, A. (1998) 'What is Agency?', American Journal of Sociology, 103(4), 962-1023.

Fairclough, N. (2005) 'Discourse Analysis in Organization Studies: The Case for Critical Realism’, Organization Studies, 26(6), 915-939.

Fowler, B. (1997) Pierre Bourdieu and Cultural Theory: critical investigations, London: Sage.

Friedland, R. and Alford, R. (1991) 'Bringing society back in: symbols, practices, and institutional contradictions', in W. Powell and P. DiMaggio (eds) The New Institutionalism in Organizational Analysis, Chicago: University of Chicago Press, 232-266.

Giddens, A. (1990) The Consequences of Modernity, Cambridge: Polity.

Hendry, J. (2000) 'Strategic decision making, discourse, and strategy as social practice', Journal of Management Studies, 37(7), 955-977.

Heracleous, L. and Hendry, J. (2000) 'Discourse and the study of organization: toward a structurational perspective', Human Relations, 53(10), 1251-1286. 
Inns, D. (2002) 'Metaphor in the literature of organizational analysis: a preliminary taxonomy and a glimpse at a humanities-based perspective', Organization, 9(2), 305-330.

Lakoff, G. and Johnson, M. (1980) Metaphors we live by, Chicago: University of Chicago Press.

Lounsbury, M., and Ventresca, M. (2003) 'The new structuralism in organizational theory', Organization, 10(3), 457-480.

McKelvey, B. (2003) 'From fields to science: can organization studies make the transition?', in R. Westwood and S. Clegg (Eds.) Debating Organization, Oxford: Blackwell, 47-72.

Mutch, A. (2005) 'Discussion of Willmott - Critical realism, agency and discourse: Moving the debate forward', Organization, 12(5), 781-786.

Outhwaite, W. (1987) New Philosophies of Social Science: realism, hermeneutics and critical theory, London: Macmillan.

Ozbilgin, M. and Tatli, A. (2005) 'Book review essay: Understanding Bourdieu's contribution to organization and management studies', Academy of Management Review, 30(4), 855-869.

Panofsky, E. (1957) Gothic architecture and scholasticism, Cleveland: Meridian.

Reed, M. (2005a) 'Reflections on the 'realist turn' in organization and management studies', Journal of Management Studies, 42(8), 1621-1644.

Reed, M. (2005b) 'Doing the loco-motion: response to Contu and Willmott's commentary on 'the realist turn' in organization and management studies', Journal of Management Studies, 42(8), 1663-1673.

Rose, S. (1993) The Making of Memory: from molecules to mind, London: Bantam. 
Rose, H. (1994) Love, power and knowledge: towards a feminist transformation of the sciences, Cambridge: Polity.

Sayer, A. (1992) Method in Social Science: a Realist Approach, London: Routledge.

Sayer, A. (2000) Realism and Social Science, London: Sage.

Scott, W. R. (2001) Institutions and Organizations, London: Sage.

Wacquant, L. J. (1993) 'On the Tracks of Symbolic Power: Prefatory Notes to Bourdieu's 'State Nobility", Theory, Culture and Society, 10, 1-17.

Watson, T. (2006) 'Review essay - The organization and disorganization of organization studies', Journal of Management Studies, 43(2), 367-382.

Whitley, R. (2003) 'From the search for universal correlations to the institutional structuring of economic organization and change: the development and future of organization studies', Organization, 10(3), 481-501.

\footnotetext{
${ }^{i}$ Unfortunately, space limits preclude us including a discussion of the work of Norman Fairclough. His 'Critical Discourse Analysis' is based on an explicitly critical realist social ontology, rejecting the tendency for the study of organization to be reduced to the study of discourse and instead locating the analysis of discourse 'within an analytically dualist epistemology which gives primacy to researching relations between agency ... and structure' (Fairclough, 2005: 916). His work is in keeping with our arguments in this paper, in that it rejects 'extreme social constructivist positions' and focuses on the relations between linguistic/semiotic elements of the social and other (including material) elements.
} 